\title{
Investigation of the $\mathrm{H}_{2} \mathrm{O}$ maser emission in $\mathrm{G} 43.8-0.1$
}

\author{
E.E. Lekht ${ }^{1,2}$ \\ 1 Instituto Nacional de Astrofísica, Óptica y Electrónica, Luis Enrique Erro No. 1, Apdo Postal 51 y 216 , 72840 Tonantzintla, \\ Puebla, Mexico \\ 2 Sternberg Astronomical Institute, 13 Universitetskij prospekt, Moscow 119899, Russia
}

Received December 8, 1998; accepted August 6, 1999

\begin{abstract}
Observations and analysis of the $\mathrm{H}_{2} \mathrm{O}$ maser emission source, associated with the star-forming region G43.8 - 0.1, are presented. The observations were carried out on the RT-22 radio telescope of the Pushchino Radio Astronomy Observatory (Russia) in 1994-1998. A new flare of emission of a group of features at radial velocities from 37.5 to $39.5 \mathrm{~km} \mathrm{~s}^{-1}$, which took place in 1996 1998 , was observed. For the $38.2 \mathrm{~km} \mathrm{~s}^{-1}$ feature, a dependence between flux density and linewidth $\left(\ln F \propto \Delta V^{-2}\right)$ was derived; it indicates that the maser is unsaturated or partly saturated.

The evolution of emission of the $42.2 \mathrm{~km} \mathrm{~s}^{-1}$ maser condensation is traced at a timespan of 16 years. The flux density was plotted versus linewidth for this feature (in the $\ln F, \Delta V^{-2}$ coordinates). This dependence contains two branches, largely shifted with respect to each other. The transition from one branch to the other took 2.5 years, while the linewidth was fluctuating. Possible causes of existence of the two branches in the maser emission evolution are discussed.

An analysis of the entire data set for 1976-1998 has shown that the time interval between the minima of integral flux in G43.8 - 0.1 was $18 \pm 1$ yr. This figure can be taken as the period of the $\mathrm{H}_{2} \mathrm{O}$ maser activity in G43.8 - 0.1.
\end{abstract}

Key words: masers — stars: formation — radio lines: stars

\section{Introduction}

The source of the $\mathrm{H}_{2} \mathrm{O}$ maser emission G43.8-0.1 (B1950 R.A. $=19^{\mathrm{h}} 9^{\mathrm{m}} 31.2^{\mathrm{s}}$, Dec. $\left.=9 \operatorname{deg} 30^{\prime} 51^{\prime \prime}\right)$ is located in a region of active star formation. It was discovered in 1976 by Genzel \& Downes (1977). The source is associated also with a compact HII region (seen in the continuum at $6 \mathrm{~cm}$ ) and with an $\mathrm{OH} 1665 \mathrm{MHz}$ maser within 10" from it

Send offprint requests to: E.E. Lekht
(Matthews et al. 1978; Evans et al. 1979). VLBI observations showed that $\mathrm{H}_{2} \mathrm{O}$ maser condensations in $\mathrm{G} 43.8-0.1$ form a shell-like structure with a radius of $\sim 0.2^{\prime \prime}$ (Downes et al. 1979). For a distance to the source of $3 \mathrm{kpc}$, this corresponds to a radius of the envelope of $\sim 10^{16} \mathrm{~cm}$.

In the catalogue of IRAS sources that are associated with a molecular outflow and/or dense molecular core G43.8-0.1 has a name of IRAS 19095+0930. Later observations of this area with a high angular resolution at 2 and $3.6 \mathrm{~cm}$ (Kurtz et al. 1994) showed that the HII region is shifted by $11.4^{\prime \prime}$ relative to the source IRAS $19095+0930$. According to Downes et al. (1979), the $\mathrm{H}_{2} \mathrm{O}$ maser source does not coincide with the HII region and is displaced by $\sim 5^{\prime \prime}$ with respect to it. Most likely, this is caused by a greater error of measurements than it was stated in the work of Downes et al. (1979). All this does not permit us to estimate the evolutionary status of the source. It is only possible to infer an age of $<10^{5}$ years (Downes et al. 1979).

Under the assumption that the positions of the maser source and ultracompact HII region do coincide, the mass and luminosity of the central star that creates the HII region G43.80-0.13 can be estimated. This can be done proceeding from the size of the envelope formed by maser spots and from the dispersion of radial velocities. In this procedure it is necessary to exclude the highestand lowest-velocity features. According to Downes et al. (1979), in this case the estimated radius of the envelope is $\sim 0.1^{\prime \prime}$, and the velocity dispersion is about $5 \mathrm{~km} \mathrm{~s}^{-1}$. The limiting mass of the star in this case is about $9 M_{\odot}$.

The observations that were carried out in 1987 at $12.2 \mathrm{GHz}$ (Koo et al. 1988) did not detect a methanol maser with a flux density $F>5 \mathrm{Jy}$. In subsequent observations of 1992-1993 a methanol maser at $12.2 \mathrm{GHz}$ was not detected with an upper limit of $0.4 \mathrm{Jy}$ (Caswell et al. 1995), but the $\mathrm{CH}_{3} \mathrm{OH}$ emission was instead revealed at another frequency, $6.6 \mathrm{GHz}$, in an interval of radial velocities of $39-44 \mathrm{~km} \mathrm{~s}^{-1}$ with a peak flux density of $152 \mathrm{Jy}$ (Menten 1991). Caswell et al. (1995) observed a 
methanol maser at the same frequency from February 1992 to September 1993. The spectrum consisted of four features. The main feature had a flux density of $140 \mathrm{Jy}$ and velocity $V_{\mathrm{LSR}}=39.5 \mathrm{~km} \mathrm{~s}^{-1}$. No flux variability of all the features of the spectrum was found.

Observations of the G43.8-0.1 region were carried out also in the lines of the CO and CS molecules (Plume et al. 1992), which yielded an estimate of the kinematic distance to the source $(3 \mathrm{kpc})$.

The high-velocity molecular gas in regions of formation of massive stars was studied by Shepherd \& Churchwell (1996). They explored the high-velocity wings of the ${ }^{12} \mathrm{CO}$ $(J=1-0)$ line for 94 sources (among them G43.8-0.1). However, they did not get an unambiguous criterion for the existence of a bipolar outflow, because high-velocity wings of the line may appear for other reasons. For instance, the cause may be numerous components, collapse, rotation, or impacts (Shepherd \& Churchwell 1996). At present nothing definite can be said about the existence of a bipolar flow in G43.8-0.1.

The peculiarity of the $\mathrm{H}_{2} \mathrm{O}$ maser source G43.8-0.1 is that, since the time of its discovery in 1976 (Genzel \& Downes 1977) and till now, emission is constantly present at $V_{\mathrm{LSR}}=42.2 \mathrm{~km} \mathrm{~s}^{-1}$ in its $\mathrm{H}_{2} \mathrm{O}$ spectrum. We have been observing the emission at this velocity all the time without interruptions (Lekht 1994, 1995). For almost 6 years (1981-1987), the intensity of this emission was growing. Then, this feature remained sufficiently stable during about 4 years. The peak flux density was within the limits of $2400-3000 \mathrm{Jy}$, and the velocity remained virtually unchanged $\left(42.20 \pm 0.05 \mathrm{~km} \mathrm{~s}^{-1}\right)$. Since 1991, the maser condensation has faded. In one year, the flux decreased twofold, and the emission again stabilized at this level $(1200-1500 \mathrm{Jy})$ till 1994.

Strong flares at different radial velocities occurred in G43.8-0.1 more or less regularly (with intervals of 1-3 years). Flare duration was from 3 months to 1.5 years, and the flux reached $2500-4550 \mathrm{Jy}$ (Lekht 1994). Most frequently, single features flared, whose intensity considerably exceeded that of blending features in the $\mathrm{H}_{2} \mathrm{O}$ spectrum. Thanks to this, such flares could be analysed as individual components. For them, we traced the variability of the flux, radial velocity and linewidth. Moreover, for two components we found a functional relationship between the variations of the flux and linewidth (Lekht 1994). From the character of this relationship, the degree of saturation of the $\mathrm{H}_{2} \mathrm{O}$ maser component can be estimated (Mattila et al. 1985; Strel'nitskij 1982).

This work is devoted to an investigation of the maser emission of the source G43.8-0.1 through the entire timespan of its observations with the aim to determine the activity period of the central star and to study variability of the emission of individual spectral components during the last flare. The results of investigation of individual components, obtained before 1994, are presented in our earlier works (Lekht 1994, 1995).

\section{Observations and data presentation}

In this work, we present spectra of maser emission of the source G43.8-0.1 in the $1.35 \mathrm{~cm} \mathrm{H}_{2} \mathrm{O}$ line from May 1994 to November 1998. We give these spectra because during this time interval a strong flare of the feature at $38.8 \mathrm{~km} \mathrm{~s}^{-1}$ took place, and the overall increase of the maser activity was observed.

The observations were carried out on the $\mathrm{RT}-22$ radio telescope of the Pushchino Radio Astronomy Observatory, Astrospace Centre of the Lebedev Institute of Physics, Russian Academy of Sciences. A cooled FET amplifier of the $22 \mathrm{GHz}$ frequency band was used. The beamwidth of the antenna at $22 \mathrm{GHz}$ is $2.6^{\prime}$. The system noise temperature was $200-300 \mathrm{~K}$. The antenna temperature of $1 \mathrm{~K}$ for an unpolarised source corresponds to a flux density of $25 \mathrm{Jy}$.

From the beginning of 1997, time intervals between consecutive observing sessions mainly did not exceed one month. This allowed us to trace the evolution of the three main parameters of spectral components during their flares: flux density, radial velocity and linewidth. In the observations, we used a 96-channel spectrum analyser with a radial-velocity resolution of $0.101 \mathrm{~km} \mathrm{~s}^{-1}$. Starting from August 1997, the spectrum analyser includes 128 channels. To investigate the linewidth of the $42.2 \mathrm{~km} \mathrm{~s}^{-1}$ feature, we used in some cases a filter bank with a frequency resolution of $2.5 \mathrm{kHz}\left(34 \mathrm{~m} \mathrm{~s}^{-1}\right.$ in the $\mathrm{H}_{2} \mathrm{O}$ line).

The $\mathrm{H}_{2} \mathrm{O}$ spectra of the source G43.8-0.1 for 19941998 are presented in Figs. 1a-g. Figure 2 shows variability of the integral flux of the emission. A sharp growth of the flux from the beginning of 1997 is caused by a series of consecutive flares of several features in the velocity interval of $37.5-39.5 \mathrm{~km} \mathrm{~s}^{-1}$.

In Fig. 3, flux density variations of the spectral features that dominated in 1994-1998 is shown. The strongest feature of this period appeared at the end of 1996 at $38.2 \mathrm{~km} \mathrm{~s}^{-1}$. At first, it was, however, strongly blended with other features; therefore, we could reliably determine its velocity only after the beginning of 1997, and the linewidth — from March 1997. The peak of the emission $(F=3750 \mathrm{Jy})$ was observed in September 1997, and then the activity rapidly faded. At the end of December 1997, the flux fell to $1200 \mathrm{Jy}$. At that time other components began to appear in the velocity interval of $37.8-39 \mathrm{~km} \mathrm{~s}^{-1}$, i.e. a cascade of less intense flares was observed.

Variations of the radial velocity and linewidth of individual features of the $\mathrm{H}_{2} \mathrm{O}$ spectrum are shown in Fig. 4 . For the component at $V_{\mathrm{LSR}}=42.2 \mathrm{~km} \mathrm{~s}^{-1}$, a smoothed curve is fitted (dashed line); it shows a small regular radial-velocity drift between early 1994 and mid-1997. Then the drift direction changed to the opposite, with a greater velocity gradient. For other features, the velocity drift was more significant.

Important linewidth variations were observed in the feature at $V_{\mathrm{LSR}}=38.2 \mathrm{~km} \mathrm{~s}^{-1}$, which flared in 1997 . 
E.E. Lekht: Investigation of the $\mathrm{H}_{2} \mathrm{O}$ maser emission in $\mathrm{G} 43.8-0.1$
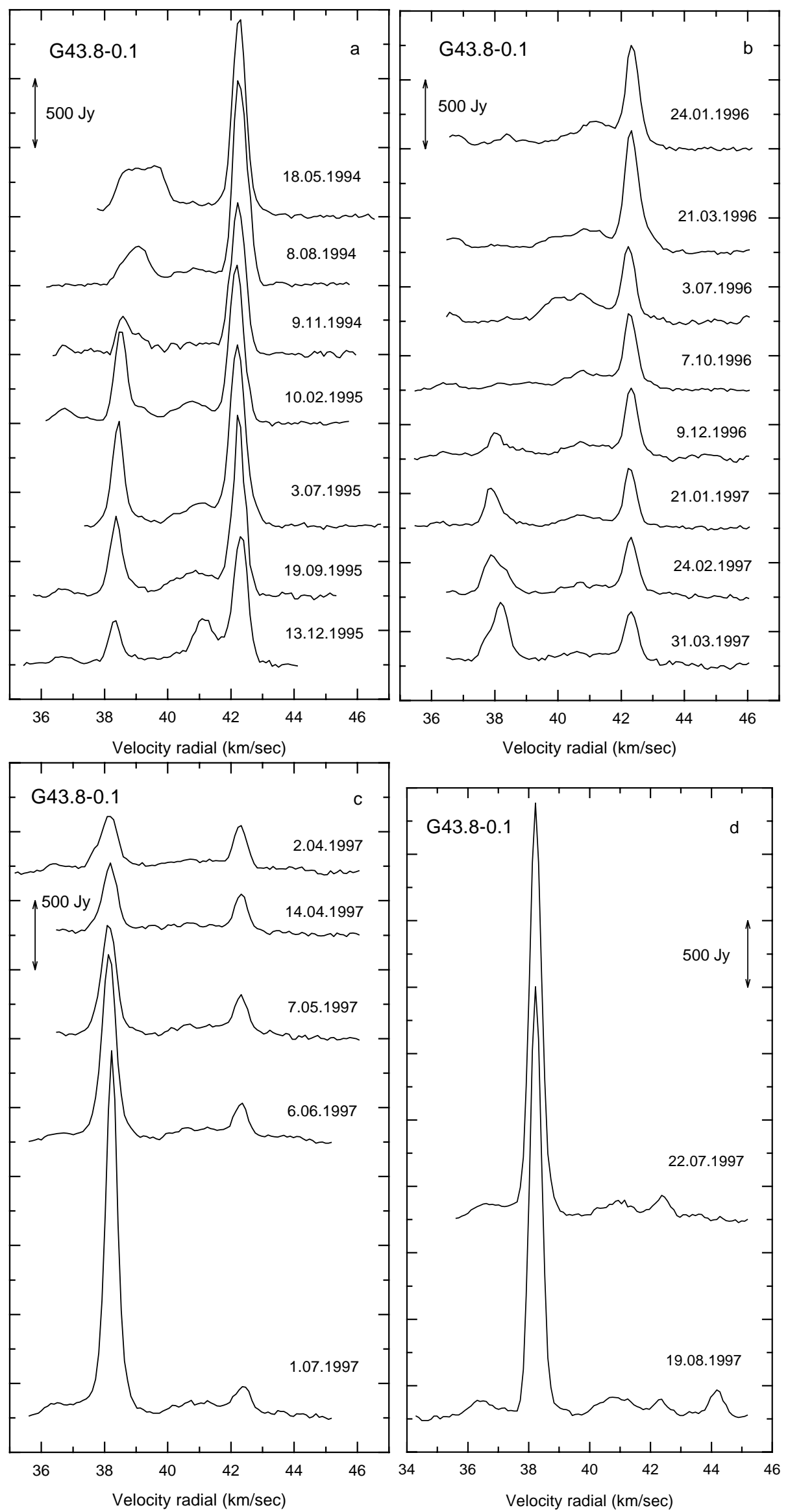

Fig. 1. a-g) Spectra of the $\mathrm{H}_{2} \mathrm{O}$ maser emission of G43.8-0.1 from May 1994 to November 1998. The vertical bar gives the flux scale 


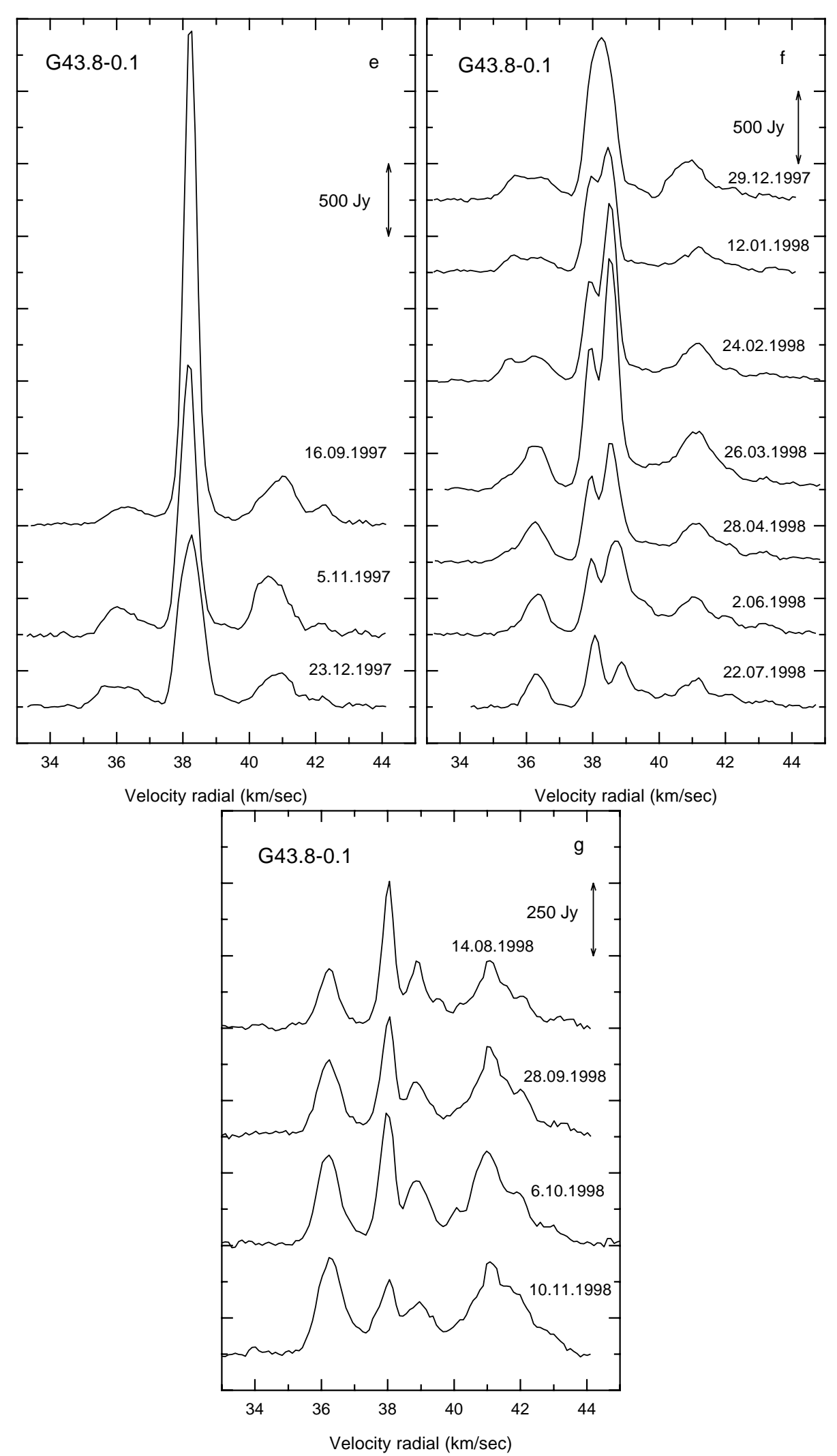

Fig. 1. continued 


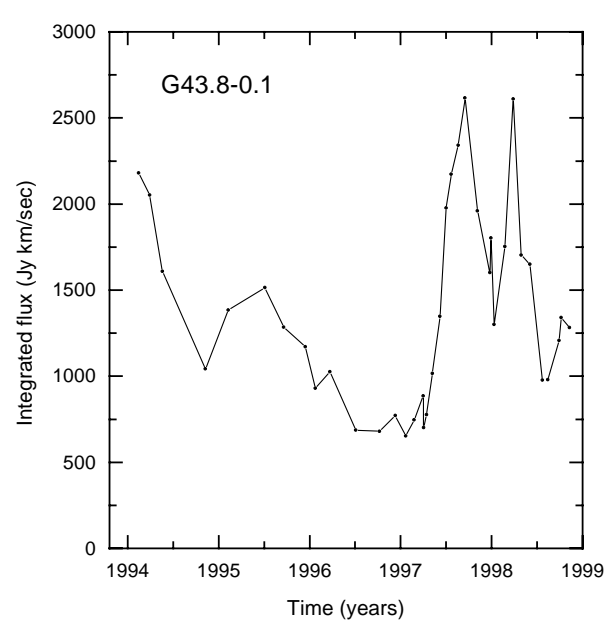

Fig. 2. Time dependence of integral flux of G43.8-0.1 in the $\mathrm{H}_{2} \mathrm{O}$ line

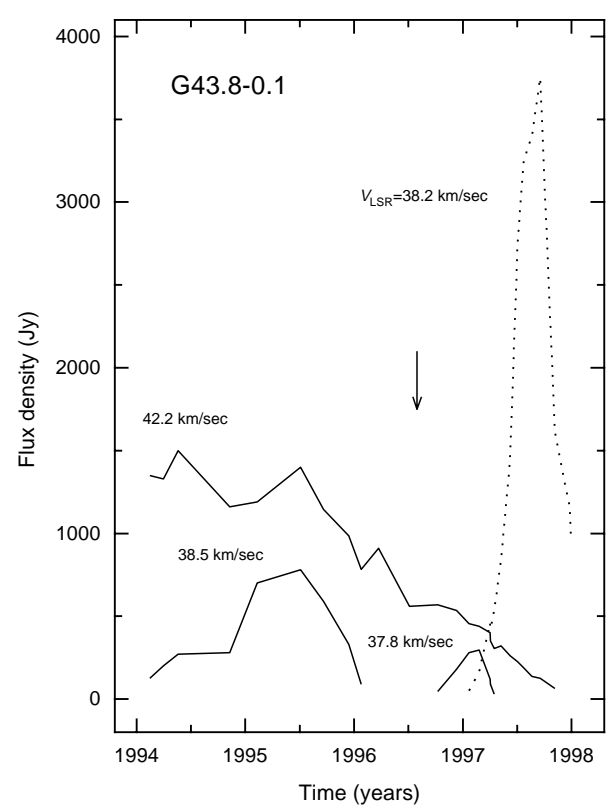

Fig. 3. Time dependence of the flux for the main components of the $\mathrm{H}_{2} \mathrm{O}$ spectra. Vertical arrow denotes the minimum of the integral flux

The degree of saturation of the maser can be evaluated from the flux-linewidth dependence, or, more conveniently, $\ln F-\Delta V^{-2}$ dependence, which expresses the logarithm of the Gaussian function. For this purpose, Fig. 5 shows the flux variability versus linewidth for the $42.2 \mathrm{~km} \mathrm{~s}^{-1}$ feature. In the case of unsaturated amplification this dependence in the $\ln F, \Delta V^{-2}$ coordinates is presented by a straight line (Elitzur 1992):

$\Delta V \propto\left[\ln F\left(V_{0}\right)\right]^{-1 / 2}$.

In the limits of the observational errors (the cross in Fig. 5) all the points fit a straight line. Only one point falls out; it corresponds in time to the onset of considerable changes of the spectrum structure. At this epoch, the linewidth is overestimated, because it was not possible to separate

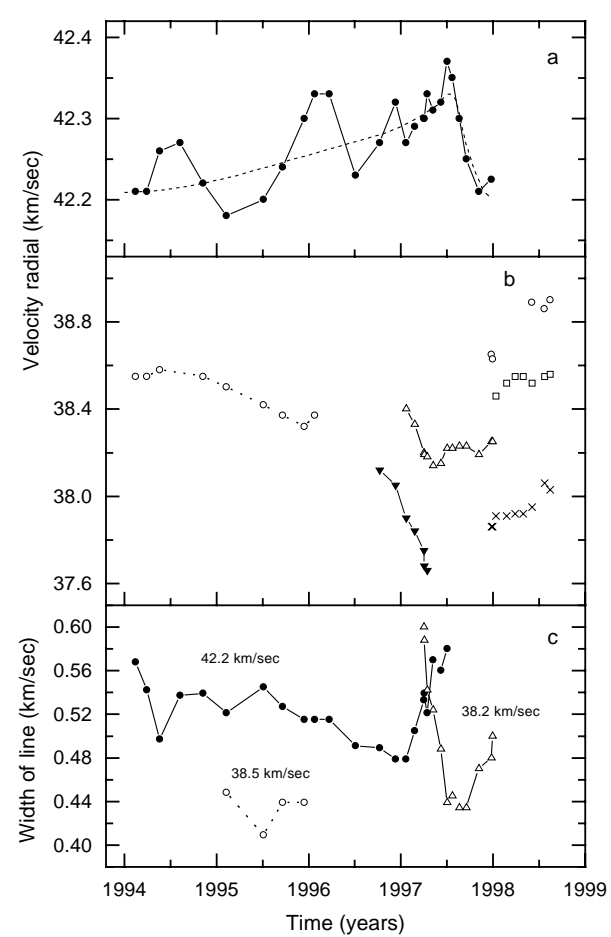

Fig. 4. Variations of radial velocity and linewidth of main $\mathrm{H}_{2} \mathrm{O}$ components of G43.8-0.1. The dashed line shows the regular radial-velocity drift of the component at $42.2 \mathrm{~km} \mathrm{~s}^{-1}$

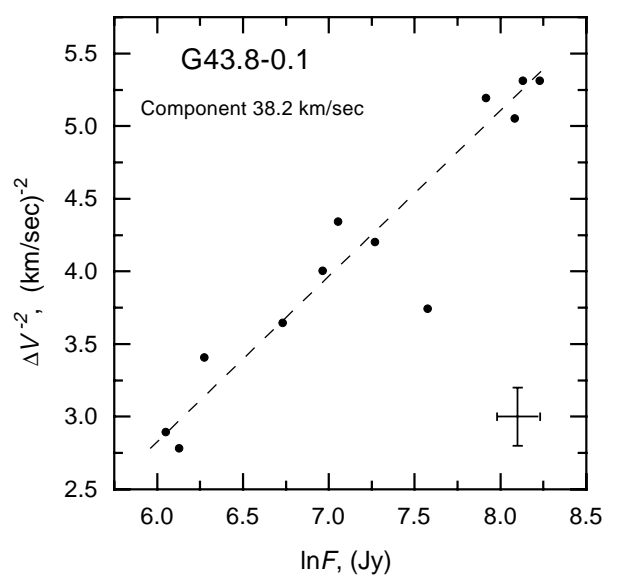

Fig. 5. Linewidth versus flux density for the component at $38.2 \mathrm{~km} \mathrm{~s}^{-1}$. The cross below shows the maximum errors of the measurements in both coordinates

the spectrum into components. The emission of the feature at $38.2 \mathrm{~km} \mathrm{~s}^{-1}$ is narrowband: at the flux maximum, the linewidth at half-power was $0.43 \mathrm{~km} \mathrm{~s}^{-1}$. Our spectral resolution $\left(0.101 \mathrm{~km} \mathrm{~s}^{-1}\right)$ was insufficient to record this line without distortions; therefore, we corrected the line for the filter passband.

On the other hand, the low-velocity wing of the line was blended by the emission of another line. However, when the main-line flux was above $500 \mathrm{Jy}$, blending was insignificant and in no way could affect the parameters of the gaussian curve fitted. Nevertheless, we corrected the 


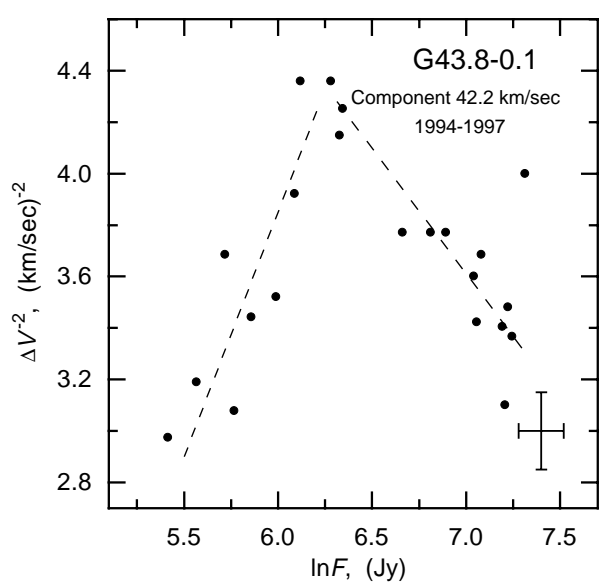

Fig. 6. Linewidth versus flux density for the component at $42.2 \mathrm{~km} \mathrm{~s}^{-1}$ in $1994-1997$

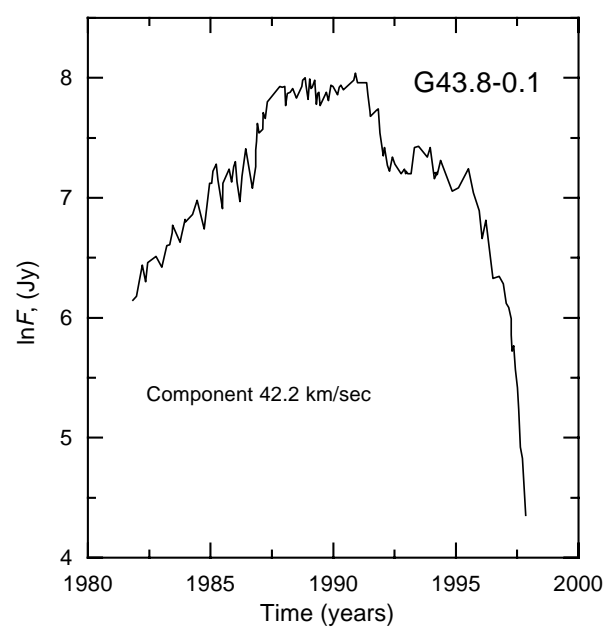

Fig. 7. Time dependence of logarithm of flux density $(\ln F)$ for the component at $42.2 \mathrm{~km} \mathrm{~s}^{-1}$ in $1981-1997$

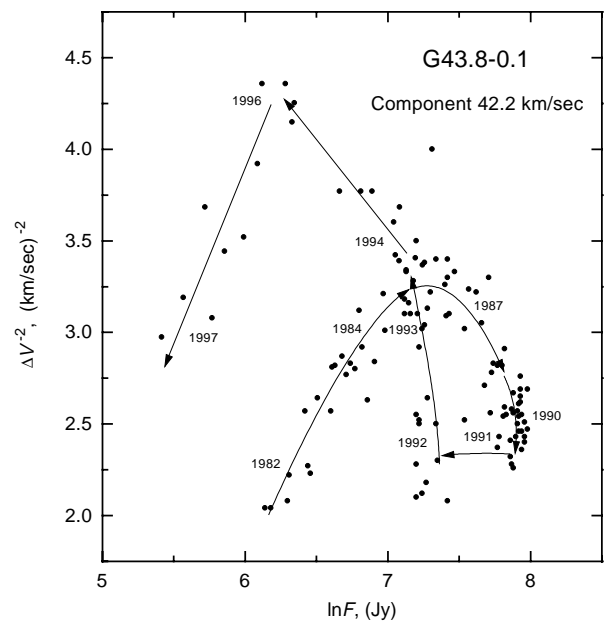

Fig. 8. Linewidth $\left(\Delta V^{-2}\right)$ versus logarithm of flux density $(\ln F)$ for the component at $42.2 \mathrm{~km} \mathrm{~s}^{-1}$ in 1981-1997. Arrows show the evolution track. Numbers denote the epochs of observations left wing of the line. Only after that we fitted a gaussian and determined its parameters, including the linewidth, whose value is most sensitive to influence of contaminating features.

Throughout the entire timespan of our observations of G43.8-0.1, for the component at $42.2 \mathrm{~km} \mathrm{~s}^{-1}$ there was a functional relation between the flux density and linewidth. At the final stage of evolution of this most "long-living" component, this functional dependence is shown in Fig. 6 . Only one point falls out. All the other points are divided into two groups, which contact near point $\Delta V^{-2} \approx 4.25$, $\ln F \approx 6.25$. Within each group, the points are mainly arranged along straight lines: $\ln F \propto \Delta V^{-2}$ for the first group and $\ln F \propto-\Delta V^{-2}$ for the second group. Note also that the second group of the points corresponds to the time interval from early 1994 to the end of 1996, while the first one refers to entire 1997.

\section{Discussion}

The $\mathrm{H}_{2} \mathrm{O}$ spectra of the source G43.8-0.1 are a superposition of emission of many components, which frequently overlap in velocity or are blended with weaker features. In our single-dish observations, it was in many cases impossible to separate such spectra into components. For this reason, we analysed only variability of the emission of the most intense features and of the integral flux.

\subsection{The flare at the radial velocity of $38.2 \mathrm{~km} \mathrm{~s}^{-1}$}

The emission at $38.2 \mathrm{~km} \mathrm{~s}^{-1}$ was present in the $\mathrm{H}_{2} \mathrm{O}$ spectrum of the source G43.8-0.1 from 1977 to 1979. Subsequent observations did not detect this emission. The emission at this velocity reappeared only at the end of 1996 in the form of a strong flare, which lasted for slightly longer than one year. In time, it happened near the activity minimum of the maser as a whole. Most probably, this flare was not local, because an increase of the emission at other radial velocities followed.

The flux density and linewidth of the flare at $38.2 \mathrm{~km} \mathrm{~s}^{-1}$ are connected by the relationship $\ln F \propto \Delta V^{-2}$ (Fig. 5). This testifies that the maser, associated with this condensation, is unsaturated or partly unsaturated.

\subsection{The maser condensation at $42.2 \mathrm{~km} \mathrm{~s}^{-1}$}

In this work, we have used the flux variability curve and the dependence between flux and linewidth variations, obtained in 1981-1993 (Lekht 1994). Figures 7 and 8 show these curves supplemented with the data obtained in 1994-1998.

We compared the two stages of evolution of the $\mathrm{H}_{2} \mathrm{O}$ maser emission at $42.2 \mathrm{~km} \mathrm{~s}^{-1}$ : from the beginning of 1982 
Table 1. The comparation of two evolutionary periods of $\mathrm{H}_{2} \mathrm{O}$ maser emission for component with $42.2 \mathrm{~km} \mathrm{~s}^{-1}$

\begin{tabular}{|c|c|c|c|}
\hline $\begin{array}{l}\text { Duration } \\
\text { (Year) }\end{array}$ & $\begin{array}{l}V_{\mathrm{LSR}} \\
\left(\mathrm{km} \mathrm{s}^{-1}\right)\end{array}$ & $\begin{array}{l}F \\
(\mathrm{Jy})\end{array}$ & $\begin{array}{l}\Delta V \\
\left(\mathrm{~km} \mathrm{~s}^{-1}\right)\end{array}$ \\
\hline $1982-1984$ & $42.30 \quad 42.15$ & 4701100 & $\begin{array}{ll}0.70 & 0.57\end{array}$ \\
\hline $1994-1996$ & $42.20 \quad 42.30$ & 1300500 & $0.55 \quad 0.48$ \\
\hline
\end{tabular}

to the end of 1984 and from the beginning of 1994 to the end of 1996. Table 1 lists the following parameters of the emission for the two stages: duration of the stage in years, radial velocity, flux and linewidth at the beginning and end of each stage. We observe similarities in the qualitative and quantitative variations of all the parameters, excluding the linewidth, which was in both cases decreasing.

In what followed, throughout 1997, the emission continued to fade, but the line was broadening, and the drift direction reversed. At the end of 1997, the intensity fell to such a level that we could reliably measure only the flux density. In 1998, the feature was very faint, and the accuracy of its parameters was insufficient to use in the corresponding plots. From July 1998, this component again started growing.

Figure 8 shows the complete evolution of the flux and linewidth of the $42.2 \mathrm{~km} \mathrm{~s}^{-1}$ feature. Arrows show the evolutionary track in time. For the same maser condensation there are two branches of the dependence between $F$ and $\Delta V$, relating to two different temporal stages of the evolution. It is obvious that the transition from one branch to the other took place between mid-1991 and early 1994. Precisely during this time interval, the linewidth was fluctuating. There was a considerable decrease of $\Delta V$ in the middle of this interval, i.e., at the beginning of 1993 (in two-month's time, $\Delta V$ decreased from 0.66 to $\left.0.55 \mathrm{~km} \mathrm{~s}^{-1}\right)$.

We can suppose that some changes happened in the region hosting the maser emission at $42.2 \mathrm{~km} \mathrm{~s}^{-1}$. This entailed oscillations of the linewidth and subsequent evolution of the emission along a different branch, which was described by functions $\ln F \propto \Delta V^{-2}$ and $\ln F \propto-\Delta V^{-2}$. The cause of this could be a transition from the saturated to unsaturated mode, a change in the maser geometry (probably due to passage of a shock) and/or some parameter of the medium, e.g. temperature. Lekht (1995) considered another possibility, namely action of the accreting matter on the given maser condensation.

In connection with our case, the latter mechanism seems less probable, because the variability of the $42.2 \mathrm{~km} \mathrm{~s}^{-1}$ emission correlates with that of the integral flux (with a phase delay of one year). The maser condensation responsible for this emission is located in the long-living emission region. This testifies to the stability of the internal geometry of this region. Most likely, its emission is stimulated by a shock wave arising from interaction of the stellar wind with the surrounding material. Such regions are most frequently associated with directional molecular outflow and radio jets inside the molecular outflow (Koo 1989; Margulis \& Snell 1989).

\subsection{Activity of the central star in G43.8-0.1}

Since 1981 we have recorded 10 flares of the emission. One of them was double-peaked, i.e. two consecutive flares took place at a radial velocity of $37.7 \mathrm{~km} \mathrm{~s}^{-1}$ in 1987 and 1989 . All the flares before April 1987 were short-living and most probably local. This assertion is substantiated by the fact that during the flares we observed no significant flux and radial-velocity variations of other features.

During the maximum activity of the entire maser (1987-1991), in addition to several strong flares, we observed a growth of the fluxes of all the features in the $\mathrm{H}_{2} \mathrm{O}$ spectrum. In this case, the flares were global, i.e. associated with an increase of activity of the maser at whole. Earlier we noted that the variability of the integral $\mathrm{H}_{2} \mathrm{O}$ flux did not bear a character of flares (Lekht 1995).

Thus, we may suppose that in G43.8-0.1 there exists some kind of equilibrium between the rate of accretion of matter onto the star, stellar luminosity variations and stellar radiation field, i.e. the process of formation of the star is more or less stationary. Nevertheless, there may exist oscillation processes with a period of the order of 10 years (Yorke \& Krügel 1977; Garlick 1978; Tutukov \& Shustov 1978), and the star formed may have maxima and minima of its activity. With regard to the earliest observations of the maser G43.8-0.1 in 1976 (Genzel \& Downes 1977) and 1977 (Genzel et al. 1979), the activity minimum of the maser was in 1978-1980. In this case, the time interval between the activity minima of the $\mathrm{H}_{2} \mathrm{O}$ maser is $18 \pm 1 \mathrm{yr}$. This figure can be taken as the period of the central-star activity in G43.8-0.1 in the process of its formation.

There can be other reasons of the observed character of the maser emission variability, for instance, shock excitation. However, the lack of direct evidence of the existence of a molecular outflow in G43.8-0.1 (Shepherd \& Churchwell 1996) and of VLA maps, obtained during our monitoring (1981-1998) does not allow an unambiguous interpretation of our data.

\section{Conclusions}

1. We have investigated the evolution of the $\mathrm{H}_{2} \mathrm{O}$ maser emission in G43.8-0.1 during a series of flares that happened at radial velocities of $37.5-39.5 \mathrm{~km} \mathrm{~s}^{-1}$ in 1996-1998. For the strongest line of this time interval, the one at $V_{\mathrm{LSR}}=38.2 \mathrm{~km} \mathrm{~s}^{-1}$, we have found that the flux density and linewidth are related as $\ln F \propto \Delta V^{-2}$. This indicates that the maser in this 
condensation is unsaturated or partly saturated. The flare at $38.2 \mathrm{~km} \mathrm{~s}^{-1}$ took place near the minimum of activity of the G43.8-0.1 maser and started a new activity cycle of the $\mathrm{H}_{2} \mathrm{O}$ maser in this source.

2. We have traced the descending branch of evolution of the $42.2 \mathrm{~km} \mathrm{~s}^{-1}$ feature. We have got a complete history of evolution of this long-living component during 16 years. The initial and final stages of the evolution turned out to be identical in duration, character of the flux and radial-velocity variability, being mirror images of each other. The only difference is that in both cases the line was narrowing (from 0.70 to $0.57 \mathrm{~km} \mathrm{~s}^{-1}$ at the initial stage and from 0.55 to $0.48 \mathrm{~km} \mathrm{~s}^{-1}$ at the final). Line narrowing, accompanied by a decrease of the flux at the descending branch of the evolution could be a consequence of a change of some parameter of the medium hosting the $42.2 \mathrm{~km} \mathrm{~s}^{-1}$ maser emission. For instance, temperature could change. The activity of this maser condensation correlated with that of the maser at whole, but always with a time delay of about one year.

3. The correlation between flux variation and linewidth is a common phenomenon in G43.8-0.1. This phenomenon, taken together with the existence of a longliving feature at $42.2 \mathrm{~km} \mathrm{~s}^{-1}$, testifies to the fact that the regions responsible for the $\mathrm{H}_{2} \mathrm{O}$ maser emission (maser condensations) in G43.8-0.1 are sufficiently stable structures. In this case, the envelope may be quite clumpy, and minor changes of the pumping conditions can considerably increase the emission intensity, provided the maser is partly unsaturated. Such stable structures can exist, for instance, in the case of the presence of a molecular outflow originating in the region of formation of a massive star.

4. The time interval between the minima of maser activity in G43.8-0.1 was $18 \pm 1$ yr. This figure can be taken as the period of the maser activity and is possible of the central-star activity in G43.8-0.1 in the process of its formation.
Acknowledgements. The author is grateful to the staff of the Pushchino Radio Astronomy Observatory for great help with the observations.

\section{References}

Caswell J.L., Vaile R.A., Ellingsen S.P., Whiteoak J.B., Norris R.P., 1995, MNRAS 272, 96

Claussen M.J., Wilking B.A., Benson P.J., Wootten A., Myers P.C., Terebey S., 1996, ApJS 106, 111

Downes D., Genzel R., Moran J.M., et al., 1979, A\&AS 79, 233

Elitzur M., 1992, Astronomical Masers. Kluwer, Dordrecht, p. 107

Evans N.J., Beckwith S., Brown R.L., Gilmore W., 1979, ApJ 227,450

Garlick A.R., 1978, A\&A 68, 113

Genzel R., Downes D., 1977, A\&AS 30, 145

Koo B.-C., 1989, ApJ 337, 318

Kurtz S., Churchwell E., Wool D.O.S., 1994, ApJS 91, 659

Lekht E.E., 1994, AZh 71, 59

Lekht E.E., 1995, AZh 72, 532

Margulis M., Snell R.L., 1989, ApJ 343, 779

Mattila K., Holsti N., Toriseva M., et al., 1985, A\&A 145, 192

Matthews H.E., Shaver P.A., Goss W.M., Habing H.J., 1978, A\&A 63, 307

Menten K.M., 1991, ApJ 380, L75

Plume R., Jaffe D.T., Evans II N.J., 1992, ApJS 78, 505

Shepherd D.S., Churchwell E., 1996, ApJ 457, 267

Strel'nitskij V.S., 1982, Pis'ma v AZh 8, 165

Tutukov A.V., Shustov B.M., 1978, Nauch. Inform. Astron. Soveta AN SSSR 41, 125

Yorke H.W., Krügel E., 1977, A\&A 54, 183 